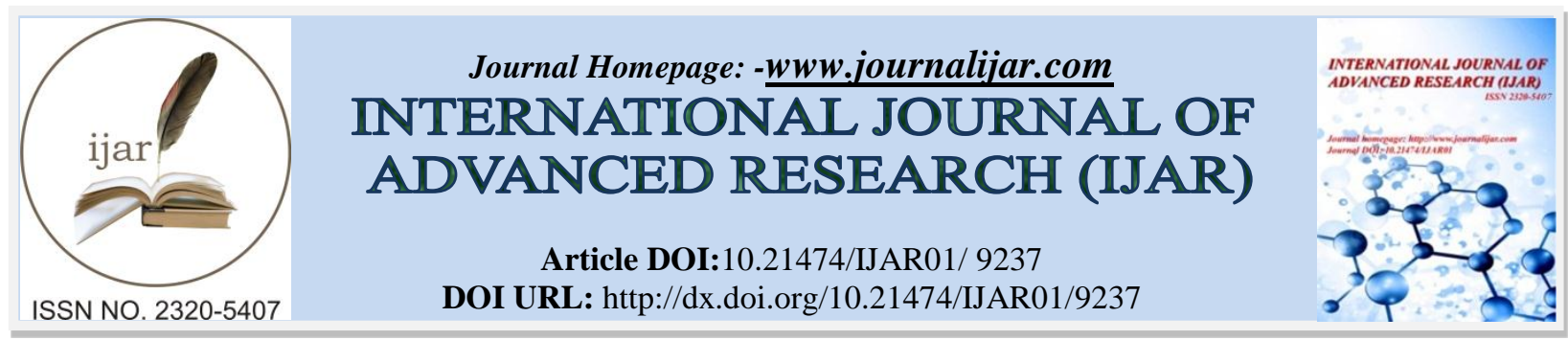

RESEARCH ARTICLE

\title{
UNEXPECTED LYMPHOMA IN AN EXPECTANT WOMAN REVEALED BY CARDIAC TAMPONADE.
}

Amal. ELAissaoui, K. Bentahar, Y. Ouharakat, I. Asfalou and E.Zbir. Cardiology center of Instruction military hospital Med V of RABAT.

\section{Manuscript Info}

\section{Manuscript History}

Received: 08 April 2019

Final Accepted: 10 May 2019

Published: June 2019

\section{Key words:-}

B-cell lymphoma; tamponade; pericardiocentesis; hemodynamic instability, swinging heart, pregnancy, mediastinal mass

\begin{abstract}
Primary mediastinal large B-cell lymphoma (PMBCL) is a distinct entity within B-cell lymphoma (2-4\% of non-Hodgkin lymphomas). It occurs most frequently in young females but rarely during pregnancy. Moreover, a cardiac tamponnade is unusually found in pregnant women. We describe a case of a 32-year-old woman, 16-week pregnant, with cardiac tamponade revealing PMBCL, where Urgent pericardiocentesis was required to maintain maternal cardiac function.

Chest-computed tomography revealed a huge mediastinal mass whose biopsy confirms the diagnosis of a PMBCL.

Spectacular improvement of clinical signs was obtained after a first line chemotherapy including rituximab, cyclophosphamide, doxorubicin, vincristine, and prednisone (R-CHOP)

Our patient received the entire chemotherapy during her pregnancy with a regular multidisciplinary follow up.

PMBCL can expose pregnant women to cardiac tamponade, and physicians should be aware of this complication mainly when pericardiocentesis is necessary.
\end{abstract}

Copy Right, IJAR, 2019,. All rights reserved.

\section{Introduction:-}

Primary mediastinal B cell lymphoma (PMBL) has been recognized as a subtype of diffuse large B cell lymphoma (DLBCL) based on its distinctive clinical and morphological features, In many patients given this diagnosis, the only site of lymphoma involvement is the mediastinum, but the lymphoma can extend locally to involve other thoracic structures. Although PMBCL frequently spreads locally from the thymus into the pleura or pericardium, it rarely turns out tamponade and uncommonly found during pregnancy.

\section{Case Report}

A 32 years old woman, 16-week pregnant, with no medical history nor cardiovascular risk factors is seen at the emergency department with shortness of breath and orthopnea, hemodynamic instability was detected with low Systolic blood pressure, tachycardia, pulsus paradoxus and Left pleural effusion syndrome. The ECG shows a micro voltage with electrical alterance and sinus tachycardia (Figure 1). Chest X ray shows a white lung aspect. (figure 2) Echocardiography performed immediately, showed pericardial effusion of great circumferential abundance with a swinging heart aspect measuring $30 \mathrm{~mm}$ facing the tip, $26 \mathrm{~mm}$ facing the lateral wall of the right ventricle with intermittent invasion of the right atrium and hemodynamic repercussion on the flow. (Figure 3)

Corresponding Author:-Amal. ELAissaoui.

Address:-Cardiology center of Instruction military hospital Med V of RABAT. 
patient was initially resuscitated with intravenous fluids and inotropic agents. "Blind" pericardial aspiration was initially performed with unsuccessful clinical result. Wheras Pericardial aspiration under transthoracic echocardiographic guidance was required, with removal of a total of $1500 \mathrm{ml}$ of citrine yellow liquid, over 3 days. afterwords repeat echocardiogram demonstrates no reaccumulation of fluid, the pigtail catheter was removed from the pericardial cavity.

MRI (Magnetic Resonance Imaging) performed later, shows Anterior mediastinal huge mass, with several connections ( pulmonary parenchyma, aorta, digestive axis ) accessible for biopsy.

This later combined with anatomopathological study demonstrated a morphological and immunohistochemical profile of Primary mediastinal large B-cell lymphoma associated with delicate interstitial fibrosis.patient was referred to a hematology department for management.

Spectacular improvement of clinical signs was obtained after a first line chemotherapy including rituximab, cyclophosphamide, doxorubicin, vincristine, and prednisone (R-CHOP). Fetal echography has showed no evidence of structural or functional diseases on the fetus. The baby had not shown any developmental delays or physical abnormalities.

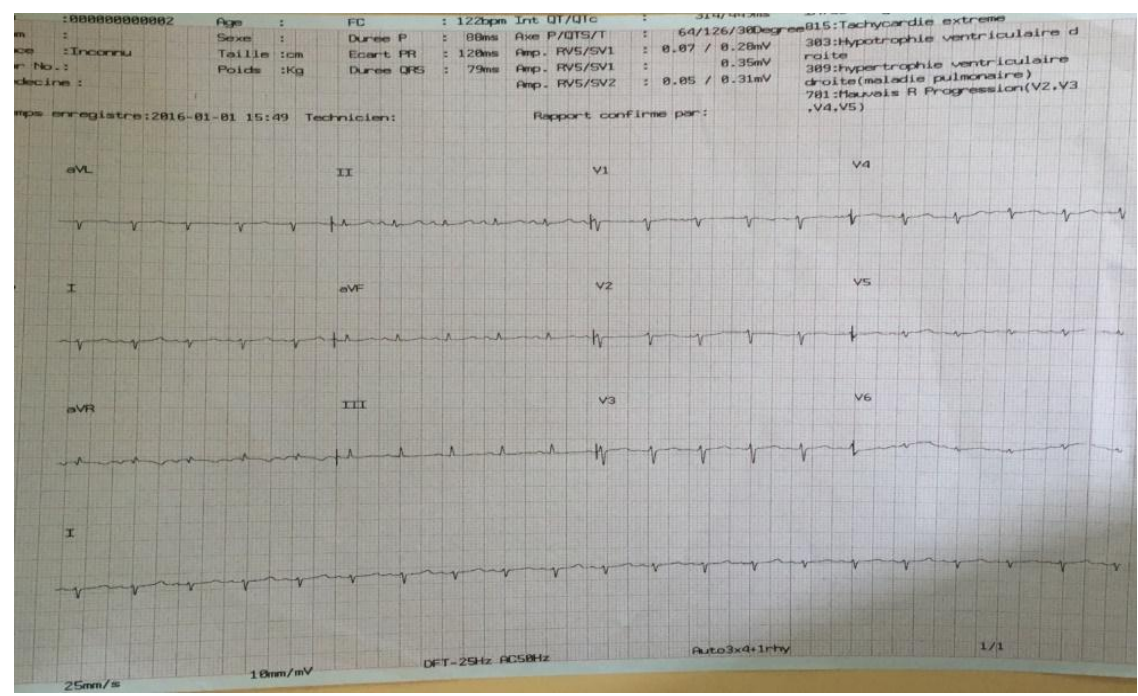

Figure 1:-Electrocardiogram : sinus tachycardia at 130 with diffuse microvoltage and electrical alterance



Figure 2:-Chest $X$ ray showed a white lung aspect. 

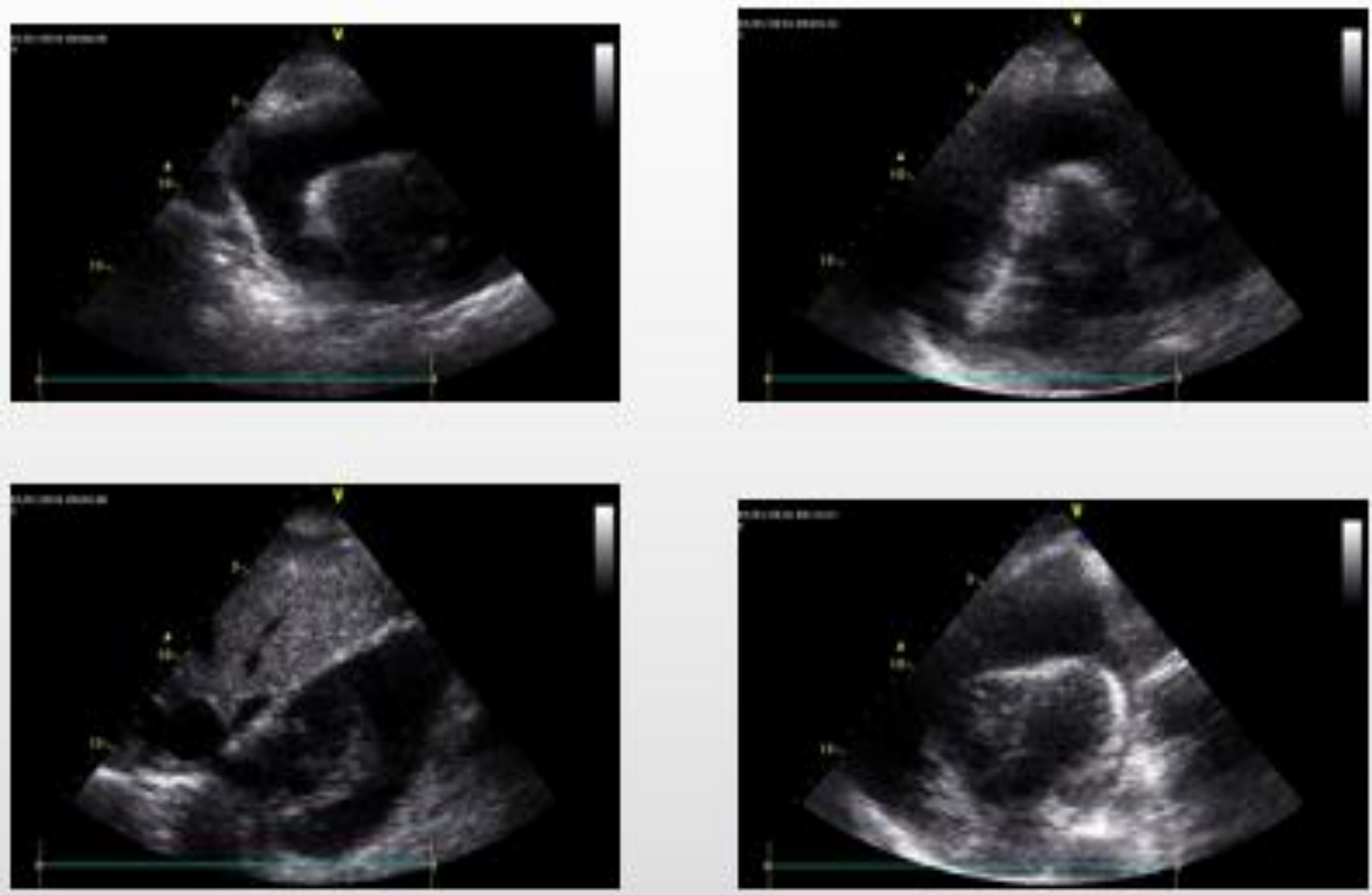

Figure 3:-transthoracic echocardiogram.where heart is seen surrounded by a very large pericardial effusion,with features of tamponade including severe collapse of the right ventricle and delated IVC

\section{Discussion:-}

Primary mediastinal (thymic) large B-cell lymphoma (PMBCL) is a subtype of diffuse large B-cell lymphoma (DLBCL) arising in the thymus. It accounts for approximately 5\% of non-Hodgkin lymphomas.[1,2,3,] First described in the 1980s, [4] as occuring in younger adults women in 30 to 40 years $[1,2,3,4]$

On the other side Insidious pericardial effusion of small abundance occurs in almost $40 \%$ of pregnant women during their third trimester of pregnancy.It is mainly due to viral or autoimmune disease;rarely do we find a neoplastic etiology. However,Inaugural presentation or evolution in cardiac tamponade is even rarer or even exceptional [5,6,7] Mediastinal lymphoma may produce a pericardial effusion, but rarely presents as cardiac tamponade [7]. The association of all those conditions: pregnancy, lymphoma, in a cardiac tamponade context, made all the authenticity of this case.

In fact, Cardiac tamponade is a medical emergency condition which classical clinical presentation is an elevated venous pressure, decreased systemic arterial pressure and a quiet heart as described in Beck's paper,long time ago. $[8]$

However, although in another study of 56 patients it has been shown that this triad is often incomplete. The major symptoms are dyspnoeato breathlessness. Physician found tachypnoea, tachycardia, pericardial rub and pulsusparadoxus. [9]

ECG might show sinus tachycardia, low voltage complexes and non-specific ST segment and T wave changes due to pericarditis. Pendular swinging motion of the heart within a pericardial effusion is manifested by Electrical alternance and it is characteristically seen only with a large effusion.[11] The ECG must be used to rule out other aetiology of hypotension rather than confirm the diagnosis of cardiac tamponade. [9].

Echocardiography is the tamponade first line of diagnosis for the positive and severity diagnosis; it can also allow the diagnosis before the installation of clinical sign[12]. It quantifies liquid effusion and appreciates the signs of 
tolerance. It can be detected initially by an abnormal septal motion, right atrial and right ventricular diastolic collapse, and reduced respiratory variation of the diameter of the inferior vena cava; TTE can also assess the distribution of the effusion [9]

Echocardiographic allow also guidance of pericardiocentesis. This later is the technique of catheter-based aspiration of pericardial fluid. It serves as a diagnostic modality via fluid analysis and a therapeutic modality especially with hemodynamic compromise [3], such as our patient case.

After acute management of this emergency, establishing An etiologic diagnosis can be a major dilemma especially in a pregnancy condition where Neoplastic pericarditisis is not common. Thuspericardial fluid cytology and pericardial/epicardial biopsy findings, CT/MRI, Increased concentrations of specific tumour markers could assess diagnosis.

The management of PMBCL involves chemotherapy and radiation. In pregnancy, therapeutic is a real dilemma, since we had limited knowledge about risks who vary with the treatment used and the term of the pregnancy of cytotoxic treatments for fetal development and on the future development during childhood. In our case, we adopt an R-CHOP (rituximab, cyclophosphamide, doxorubicin, vincristine, and prednisone) regimen who achieve a cure rate of $82 \%$ in literature $[13,14]$.

In fact, this therapy allowed a spectacular improvement of clinical signs. The patient had received the entire chemotherapy during her pregnancy with a regular multidisciplinary follow up. Delivery was uneventful.

\section{Conclusion:-}

Cardiac tamponade is a life-threatening emergency; mortality might be higher in pregnancy, requiring immediate pericardiocentesis. Neoplastic etiology is not common. We highlights in this case that PMBCL can exposes to cardiac tamponade, that Pericardiocentesisis is the first line therapy and the fact that managing pregnant patients with hematological tumors pose more conflicts.

Since A multidisciplinary approach, including oncologists, radiologists, obstetricians, cardiologist and psychiatrist specialists, social services, is mandatory to optimize management

\section{References:-}

1. Charles Blake Hutchinson, MD; Endi Wang, MD, PhD. Primary Mediastinal (Thymic) Large B-Cell Lymphoma A Short Review With Brief Discussion of Mediastinal Gray Zone Lymphoma . Arch Pathol Lab Med-Vol 135, 394-398, March 2011

2. Cazals-Hatem D, Lepage E, Brice P, et al. Primary mediastinal large B-cell lymphoma: a clinicopathologic study of 141 cases compared with 916 nonmediastinal large B-cell lymphomas, a GELA ("'Grouped'Etude des Lymphomes de l'Adulte'') study. Am J SurgPathol. 1996;20(7):877-888.

3. Barth TF, Leitha“ user F, Joos S, et al. Mediastinal (thymic) large B-cell lymphoma: where do we stand? Lancet Oncol. 2002;3(4):229-234. 3.

4. Lichtenstein AK, Levine A, Taylor CR, et al. Primary mediastinal lymphoma in adults. Am J Med. 1980;68(4):509-514.

5. Ristić, A., Seferovic, P., Ljubic, A. et al. Heart (2003) 28: 209. Https://doi.org/10.1007/s00059-003-2470-3

6. Raúl A. Montañez-Valverde1,3, †*, Neal Ivan Olarte2, $\uparrow$, Gerardo Zablah2, David Hurtado-de-Mendoza2 and Rosario Colombo. Swinging heart caused by diffuse large B-cell lymphoma .Oxford Medical Case Reports, 2018;9, 299-301

7. Refaat MM, Katz WE. Neoplastic pericardial effusion. ClinCardiol 2011;34:593-8.

8. Beck CS. Two cardiac compression triads. JAMA 1935;104:714-716.

9. D. COLLINSAetiology and Management of Acute Cardiac Tamponade. Critical Care and Resuscitation 2004; 6: 54-58)

10. Guberman BA, Fowler NO, Engel PJ, Gueron M, Allen JM. Cardiac tamponade in medical patients. Circulation 1981;64:633-640.

11. Hancock EW. Cardiac Tamponade. Med Clin N Am 1979;63:223-237.

12. Kronzon I, Cohen ML, Winer HE. Contribution of echocardiography to the understanding of the pathophysiology of cardiac tamponade. J Am CollCardiol 1983;4:1180-1182. 
13. Khalid S, Khalid A, Daw H, et al. (February 21, 2018) Management of Primary Mediastinal B-Cell Lymphoma in Pregnancy. Cureus 10(2): e2215. DOI 10.7759/cureus.2215

14. Perez CA, Amin J, Aguina LM, Lavina MC, Santos ES: Primary mediastinal large B-cell lymphoma during pregnancy. Case Rep Hematol. 2012, 2012:197347. 10.1155/2012/197347. 